\title{
Transport time to trauma facilities in Karachi: an exploratory study
}

\author{
Roomasa Channa • Hira Altaf Jaffrani • \\ Aamir Javed Khan - Talal Hasan • \\ Junaid Abdul Razzak
}

Received: 23 January 2008 / Accepted: 20 July 2008 / Published online: 24 September 2008

(C) Springer-Verlag London Ltd 2008

\begin{abstract}
Background Rapid urban growth in developing countries has outpaced the development of health infrastructure, including trauma centers, leading to potential delays in trauma care. This study was conducted in Karachi, a city of 16 million people in Pakistan.

Aims Our aim was to determine the time taken to reach the nearest 24-h emergency care facility (ECF) and the government-designated trauma center (TC). We also sought to determine the availability of supplies and equipment required for "basic" trauma care at these centers.

Methods We selected five towns in Karachi that had the highest number of road traffic injuries (RTIs) (as identified through medicolegal records). We then measured the time taken to reach the nearest ECF and the government-
\end{abstract}

R. Channa $\cdot$ H. A. Jaffrani

Aga Khan University,

Karachi, Pakistan

\section{A. J. Khan}

Interactive Research \& Development,

Karachi, Pakistan

\author{
A. J. Khan \\ Johns Hopkins Bloomberg School of Public Health, \\ Baltimore, MD, USA \\ T. Hasan \\ London School of Economics, \\ London, UK

\section{J. A. Razzak ( $\square)$} \\ Section of Emergency Medicine, Department of Medicine, \\ Aga Khan University, \\ Stadium Road, P.O. Box 3500, Karachi 74800, Pakistan \\ e-mail: junaid.razzak@aku.edu
}

designated TC from four compass points within each town. We also asked about the equipment and supplies used in basic trauma care.

Results All three TCs in Karachi were located in the selected towns and were within $5.0-10.5 \mathrm{~km}$ of each other. The transport times to the 3 TCs were an average of 13.3 $\min ( \pm 7.1)$ and to the 16 ECFs an average of $4.7 \mathrm{~min}( \pm 2.4)$ ( $p$ value $<0.00$ ). Most ECFs did not have all equipment and supplies necessary for basic trauma care; $90 \%$ had the basic equipment for management of airway, oxygen, and IV fluids, $70 \%$ had morphine, and $45 \%$ had C-spine collars. Conclusions Vital time is lost in reaching a governmentdesignated TC. ECFs might be an alternative option, but are not fully equipped and funded to provide adequate trauma care to all.

Keywords Emergency care · Trauma care · Travel timing · Pakistan

\section{Introduction}

Road traffic injuries (RTIs) are responsible for approximately 1.2 million deaths worldwide and 50 million injuries each year [1]. RTI rates are particularly high in the developing world due to poorly maintained road networks and motor vehicles [2]. These rates are projected to rise as developing countries become increasingly motorized [3]. Timely provision of emergency medical care can significantly reduce morbidity and mortality [2] and also plays a significant role in post-injury outcome [4]. Rapid urban growth in developing countries has outpaced the development of health infrastructure, including trauma centers, leading to potential delays in trauma care. One possible 
Table 1 Times to TCs and ECFs

\begin{tabular}{lllll}
\hline Variable & Mean $( \pm \mathrm{SD})$ & Quartiles & \\
\cline { 2 - 4 } & & 25 & 50 & 75 \\
\hline Time taken to reach TC, $\min (n=24)$ & $13.3( \pm 2.5)$ & 8 & 11 \\
Time taken to reach ECF, $\min (n=16)$ & $4.3( \pm 2.4)$ & 3 & 4 \\
\hline
\end{tabular}

TCs Trauma centers, ECFs emergency care facilities

solution is to identify and designate other emergency care facilities (ECF) as lower level trauma facilities to receive trauma patients with less severe injuries. Many such facilities are not prepared to take care of trauma because of lack of equipment or government incentives.

Besides existence of facilities, the availability of adequate transport is essential. An absence of emergency medical transport may become a barrier to care in developing countries. In Guinea-Bissau, 20 of 125 acutely ill children died either on their way to hospital or while waiting in the reception area of an outpatient clinic [5]. In Malaysia, a team assessing the value of the risk coding system in pregnancy concluded that better communications, a more effective transport system, and better emergency care in hospitals were needed in order to reduce maternal mortality [6]. Karachi is a city with a population of 16 million. It has three government-designated trauma centers (TC) and numerous 24-h ECF. Ambulance services are available in the city - the largest one being provided by Edhi. It operates 75 ambulances from 35 centers across the city. Studies show that ambulances are not commonly used by the people of Karachi. Taxi cabs and private cars are the commonly used modes of transport [7].

We set out to determine the time taken to reach a government-designated TC from the areas with the highest numbers of RTIs compared to an alternative 24-h ECF. We

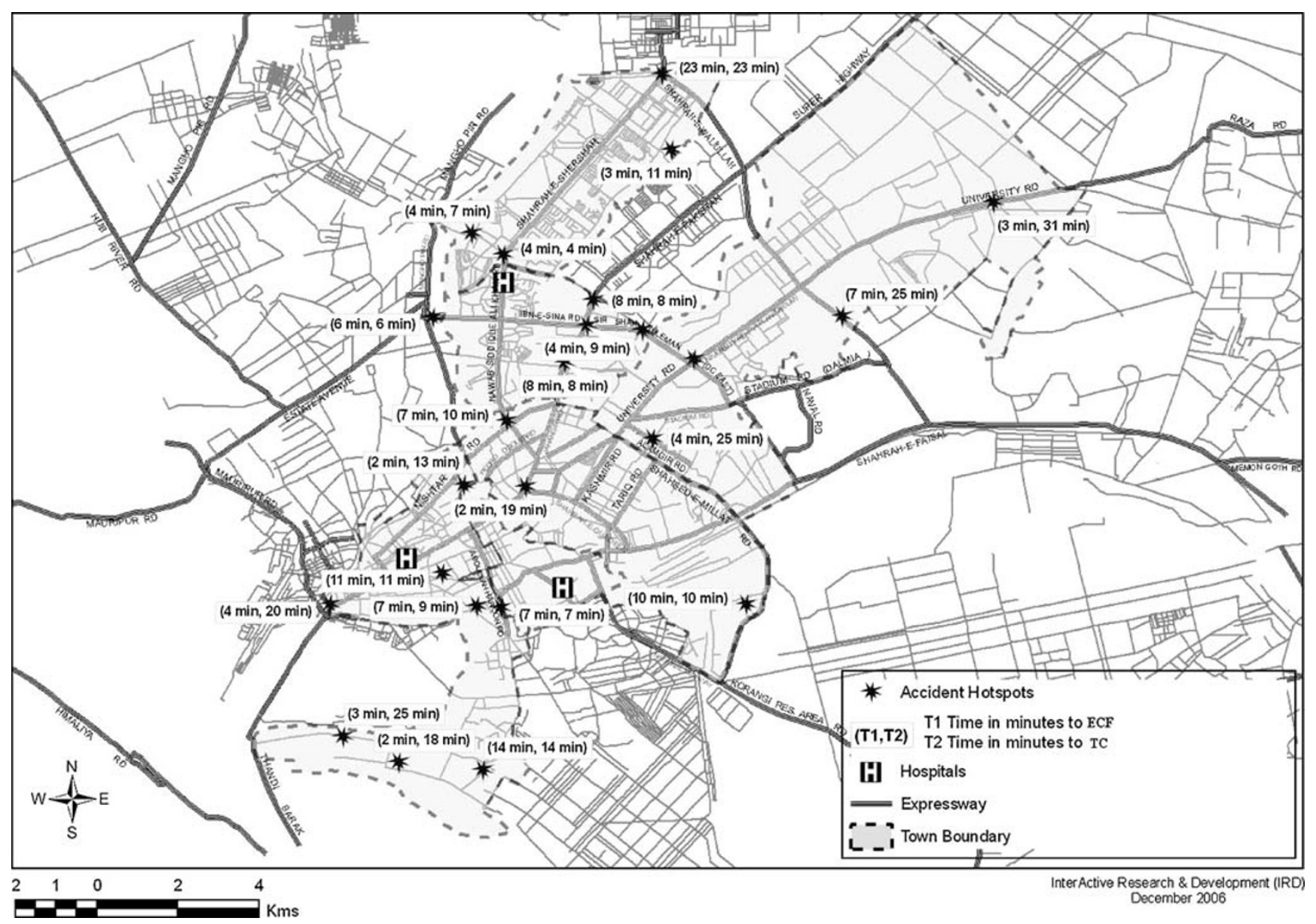

Fig. 1 Map: time to way stations and major trauma centers from select accident hot spots in Karachi, Pakistan, 2006 
also sought to determine whether the ECFs had the supplies and equipment required for basic trauma care.

\section{Methods}

This was a cross-sectional study conducted in Karachi, Pakistan. There are only three major government-designated TCs for the 16 million residents of Karachi, which will be the sixth most populous city in the world by 2015 [8]. Medicolegal departments of the three TCs were asked for permission to access their database. Karachi is divided into 18 administrative units called "towns." We identified five towns as the ones from where $50 \%$ of the total RTIs were reported. Four points were selected from the northern, southern, eastern, and western extremes of each town. The fifth town, being larger than the others, was first divided into two halves after which the four compass points were selected. A private car with a professional driver was used between 1300 and 1700 hours to travel from the selected point to the nearest TC used by the people of that town. The reason for using a private car was that taxi cabs and private cars are the most commonly used modes of transport by emergency victims in our setting. Ambulances are rarely used [7]. Two stopwatches were used to measure the time-one for time taken to travel to the nearest ECF and the second one for the time taken to reach the government-designated TC. The second part of the study involved determining the availability of basic trauma care at the ECFs where a given patient could have been taken on his way to TCs. We administered the questionnaire to the most senior doctor in charge of the emergency department at the ECF. This questionnaire asked about the availability of basic items required for management of circulation and shock, airway insufficiency, and spinal injury, for diagnosis and monitoring, safety for health care personnel, pain control, and medicines. Each item in the questionnaire was rated according to the following scoring system: 0 if absent or present only sometimes and 1 if present at all times.

\section{Results}

The area included in our study was $121.7 \mathrm{~km}^{2}$ and had a population of 3,109,192 representing 3\% of Karachi's land area, $20 \%$ of Karachi's population, five towns, and all three major government-designated TCs. We took a total of 24 points for determining travel times. For all 24 we have readings to the $\mathrm{TC}$, and for 16 we have readings to the nearest ECF. For the remaining 8 points, the TC was the nearest health care facility; therefore, we do not have ECF readings for those points.
The time taken to reach the TC from the selected points ranged from a minimum of $4 \mathrm{~min}$ to a maximum of $31 \mathrm{~min}$. The distance from a landmark to the nearest major TC ranged from $1 \mathrm{~km}$ to $10.6 \mathrm{~km}$. The average time taken to reach the nearest $\mathrm{TC}$ was $13.3 \mathrm{~min}$. The average time taken to reach the nearest ECF was 4.7 min (see Table 1 and Fig. 1). The difference in times was significant with a $p$ value of $<0.005$ (95\% confidence interval: 4.86-12.3 $\mathrm{min}$ ).

The staff at 13 ECFs were interviewed. Most (almost $90 \%$ ) had the basic equipment for management of airway, oxygen supply, and IV fluids. Of 13, 9 (70\%) had pain medications such as morphine and only $6(45 \%)$ possessed a cervical spine collar (see Table 2).

\section{Discussion}

The city has only three TCs and travel times are over 30 min within a maximum distance of $10 \mathrm{~km}$ from the TC. For distant areas in the city (i.e., the remaining 97\% of the area covered), travel times to these TCs will be much higher. The city has a poor road infrastructure and public transport vehicles are prone to frequent accidents [9].

The ambulances are staffed by drivers with no training in emergency trauma care or basic life support. The ambulances are typically equipped with a radio and a stretcher

Table 2 Availability of supplies at ECFs

\begin{tabular}{ll}
\hline Equipment & $\begin{array}{l}\% \text { of small ECFs } \\
\text { having the item }(n=13)\end{array}$ \\
\hline Oral or nasal airway & 100 \\
Laryngoscope & 92 \\
Endotracheal tube & 92 \\
Bag-valve-mask & 92 \\
Oxygen supply (cylinder, concentrator, & 100 \\
or other source) & \\
Crystalloid & 92 \\
Colloids & 92 \\
Intravenous infusion set (lines and & 92 \\
cannulas) & \\
External pressure for bleeding & 92 \\
Basic immobilization (sling, splint) & 100 \\
Spine board & 46 \\
Immobilization: C-collar, backboard & 46 \\
Tetanus prophylaxis (toxoid, antiserum) & 92 \\
Oxygen & 100 \\
Morphine (or equivalent) & 69 \\
Normal saline solution (0.9\% isotonic) & 85 \\
Urinary catheter with collection bag & 92 \\
Plain films & 92 \\
Gloves & 92 \\
Gowns & 80 \\
Sharps disposal & 69 \\
\hline
\end{tabular}


and in some cases with an oxygen tank, a suction machine, and a "first aid box" for wound dressing. The drivers maintain contact with the ambulance service control room. However, they have no communication with the hospital and do not follow any treatment protocols.

Usually, a bystander at the scene of the emergency calls the ambulance service. The dispatch center then instructs the ambulance service nearest to the site to send an ambulance. The ambulance then picks up the patient and transports him or her to the nearest TC [10].

Newspapers frequently report deaths of trauma victims due to delay in accessing emergency care. Our results, surprisingly, show travel times that are much lower than we expected at the outset of our study. The reason could be the variability in traffic at different times of the day. Our study was carried out between 1300 and 1700 hours on working days. It is very likely that the travel time will be different at other times of the day and on weekends.

The level of medical facilities is an important factor affecting the number of people killed in road traffic accidents, which cost Pakistan about $£ 260$ million per annum [11]. Until recently, trauma patients were exclusively taken to government-designated TCs with a medicolegal department. New legislation allows ECFs to provide services to RTI victims. This means that there are more hospitals available to cater to the needs of trauma patients and are on an average $4.5 \mathrm{~min}$ away from any given location. However, essential items such as cervical spine collar, spine board, and pain medications such as morphine are not universally available at the ECFs. In addition, most ECFs are privately owned and charge a fee for services. This becomes an important issue where more than $70 \%$ of the population earns less than $\$ 2$ per day [12]. The true usefulness of ECFs will become apparent only after they become better equipped and receive incentives to provide trauma care. Efforts need to be made to upgrade trauma care services in the city.

There are a number of limitations in our exploratory study. We were able to determine timings only at one particular time of the day. We are therefore unable to comment on seasonal, monthly, off-peak, and peak hour variability.

We relied on the response of the doctors in the ECFs regarding the presence of supplies at all times and did not check and confirm the inventory of the ECFs at that time. Further studies need to be done to determine the differences in patient outcome depending on whether they use an ECF or TC and to establish guidelines for the appropriate referral, transfer, and management plans for different patients.

\section{Conclusion}

The present system in our city only has level 4 tTCs that are the three TCs. In order to establish an organized trauma system, hospitals other than designated major TCs need to be upgraded to receive and treat less acutely ill patients. Hence, there is a need for lower level TCs. Our study shows that ECFs are located at sites that can be reached within convenient times from accident hot spots and may be able to serve as such if equipped adequately.

Conflicts of interest None.

\section{References}

1. Cole TB (2004) Global road safety crisis remedy sought: 1.2 million killed, 50 million injured annually. JAMA 291(21):25312532

2. Sasser SVM, Kellermann A, Lormand JD (2005) Prehospital trauma care systems. World Health Organization, Geneva

3. Peden MM, McGee K, Krug E (2002) Injury: a leading cause of the global burden of disease, 2000. Department of Injuries and Violence Prevention, Noncommunicable Diseases and Mental Health Cluster, World Health Organization, Geneva

4. Mock CN, Adzotor KE, Conklin E et al (1993) Trauma outcomes in the rural developing world: comparison with an urban level I trauma center. J Trauma 35(4):518-523

5. Sodemann M, Jakobsen M, Mølbak K et al (1997) High mortality despite good care-seeking behaviour: a community study of childhood deaths in Guinea-Bissau. Bull World Health Organ 75 (3):205-212

6. Geefhuysen CJ, Isa AR, Hashim M et al (1998) Malaysian antenatal risk coding and the outcome of pregnancy. J Obstet Gynaecol Res 24(1):13-20

7. Razzak JA, Cone DC, Rehmani R (2001) Emergency medical services and cultural determinants of an emergency in Karachi, Pakistan. Prehosp Emerg Care 5(3):312-316

8. United Nations Department of Economic and Social Affairs/ Population Division (UNDESA) (2004) World population prospects. United Nations, New York

9. Khan S (2007) Transport poor Karachi. Department for International Development, London. Available from: http://www.ucl. ac.uk/dpu-projects/drivers_urb_change/urb_infrastructure/pdf transport/DFID Sohail Khan\% $\%$ Transport \%20Poor Karachi. pdf. Cited 24 Dec 2007

10. Razzak JA (2005) The potential of ambulance records for a road traffic safety agenda in low-income cities: studies from Karachi, Pakistan. Dissertation, Karolinska Institute, Stockholm

11. Baguley CJ, Jacobs GD (2000) Traffic safety issues for the next millennium. Available from: http://www.transport-links.org/ transport_links/filearea/publications/1_769_PA3642.pdf. Cited 24 Dec $200 \overline{7}$

12. World population data sheet (2006) Population Reference Bureau, Washington, DC. Available from: http://www.prb.org/pdf06/ 06WorldDataSheet.pdf. Cited 24 Dec 2007 